\title{
Hamiltonian approach to Coulomb gauge Yang-Mills Theory
}

\section{H. Reinhardt ${ }^{* i}$}

Institut für Theoretische Physik, Auf der Morgenstelle 14

D-72076 Tübingen, Germany

E-mail: h.reinhardt@uni-tuebingen.de

\section{W. Schleifenbaum}

Institut für Theoretische Physik, Auf der Morgenstelle 14

D-72076 Tübingen, Germany

\section{Epple}

Institut für Theoretische Physik, Auf der Morgenstelle 14 D-72076 Tübingen, Germany

\section{Feuchter}

Institut für Theoretische Physik, Auf der Morgenstelle 14

D-72076 Tübingen, Germany

The vacuum wave functional of Coulomb gauge Yang-Mills theory is determined within the variational principle and used to calculate various Green functions and observables. The results show that heavy quarks are confined by a linearly rising potential and gluons cannot propagate over large distances. The 't Hooft loop shows a perimeter law and thus also indicates confinement.

The XXV International Symposium on Lattice Field Theory

July 30-4 August 2007

Regensburg, Germany

\footnotetext{
${ }^{*}$ Speaker.

${ }^{\dagger}$ This work was supported by the Deutsche Forschungsgemeinschaft (DFG) under contract no. Re856/6-1,2.
} 


\section{Introduction}

The confinement puzzle has been with us ever since the birth of quantum chromodynamics (QCD). By means of lattice calculations, it has been possible to penetrate the infrared nonperturbative sector of QCD and recover a confining potential between (static) quarks [1]. At present, however, available lattice sizes do not suffice to describe the Green functions in the deep infrared [2].

The continuum approach, on the other hand, has the intriguing feature that the infrared limit can be studied asymptotically. In the last decade, a new understanding of infrared QCD has arisen from studying continuum Yang-Mills (YM) theory via Dyson-Schwinger equations. The Landau gauge has the advantage of being covariant and therefore encouraged many to intensive investigation of the infrared properties of YM theory [3, 4]. In Coulomb gauge, non-covariance brings about severe technical difficulties which are only recently on the verge of being overcome [5]. Nevertheless, the Coulomb gauge might be the more efficient choice to identify the nonabelian degrees of freedom. It is well-known that screening and anti-screening contributions to the interquark potential are neatly separated in Coulomb gauge perturbation theory [6]. As for the infrared domain, the Gribov-Zwanziger scenario serves as a transparent confinement mechanism [7, 8].

A further advantage of working in the physical Coulomb gauge is that one may pass over to a Hamiltonian description. This opens up direct access to the heavy quark potential via the expectation value of the Hamiltonian. In recent years, variational methods have been pursued to solve the Yang-Mills Schrödinger equation with a Gaussian type of wave functional $[9,10,11$, 12]. Despite Feynman's critique [13], it turns out that the wave functional is sensitive to infrared modes and the variational method a powerful tool, at least for the qualitative description of YM theory. With careful treatment of the operator ordering in Coulomb gauge [14], it is possible to find a strictly linearly rising heavy quark potential. We report on the latest results found in the Hamiltonian approach to YM theory in Coulomb gauge. This includes the full calculation of gluon and ghost Green functions and a running coupling. Furthermore, the 't Hooft loop, an (dis-)order parameter for confinement, will be calculated using the results of the Green functions.

This paper is organized as follows. In section 2, the Yang-Mills Hamiltonian in Coulomb gauge and the equations of motion are introduced. The latter will be solved variationally and the heavy quark potential and the running coupling are presented in section 3. The 't Hooft loop is discussed in section 4 and conclusions are given in section 5 .

\section{Yang-Mills Schrödinger equation and Dyson-Schwinger equations}

In the canonical quantization approach, we choose $A_{0}^{a}(x)=0$ and impose the usual equal-time commutation relations among the gauge field $A_{i}^{a}(x)$ and the conjugate momentum $\Pi_{i}^{a}(x)$ to arrive at the Weyl gauge Hamiltonian. Since $A_{0}$ originally serves as the Lagrange parameter of the Gauss law, the choice of Weyl gauge requires a restriction on the Hilbert space,

$$
\hat{D} \Pi|\Psi\rangle=-g \rho_{m}|\Psi\rangle
$$

where $g$ is the gauge coupling, $\rho_{m}^{a}(x)$ the density of external color charges, and $\hat{D}_{i}^{a b}=\partial_{i} \delta^{a b}+g \hat{A}_{i}^{a b}$ with $\hat{A}_{i}^{a b}=A_{i}^{c} f^{a c b}$. Fixing the residual time-independent local gauge invariance by the Coulomb 
gauge, $\partial_{i} A_{i}=0$, and eliminating the longitudinal part of the momentum operator $\Pi$ by means of the Gauss law (2.1), one arrives at the Hamiltonian that depends only on transversal fields,

$$
H=\frac{1}{2} \int\left(\mathscr{J}^{-1} \Pi \mathscr{J} \Pi+B^{2}+g^{2} \mathscr{J}^{-1} \rho F \mathscr{J} \rho\right) .
$$

The appearance of the Faddeev-Popov determinant $\mathscr{J}[A]=\operatorname{det}(-D[A] \partial)$ is due to a non-trivial change of coordinates to the transverse fields and turns out to be crucial to the infrared properties of the theory. The latter term in Eq. (2.2) describes the Coulomb interaction of dynamical and external charges $\rho=-\hat{A} \Pi+\rho_{m}$ via

$$
F^{a b}(x, y)=\left\langle x, a\left|(-D \partial)^{-1}\left(-\partial^{2}\right)(-D \partial)^{-1}\right| y, b\right\rangle
$$

and reduces to the familiar Coulomb law in the abelian theory.

With the Hamiltonian (2.2) at hand, we may apply the variational principle to find the wave functional $\Psi[A]=\langle A \mid \Psi\rangle$. Inspired by QED, we choose [10]

$$
\Psi[A]=\frac{\mathscr{N}}{\sqrt{\mathscr{J}}} \int \mathscr{D} A \exp \left(-\frac{1}{2} \int A \omega A\right)
$$

with a normalization constant $\mathscr{N}$. The factor of $\mathscr{J}^{-1 / 2}$ is chosen to alleviate the computation of expectation values, similar to defining radial states in quantum mechanics. A different power of $\mathscr{J}$ in the wave functional does not change the properties of the solution [15]. One may think of the variational parameter $\omega$ as in the inverse of the gluon propagator,

$$
D_{i j}^{a b}(x, y)=\left\langle\Psi\left|A_{i}^{a}(x) A_{j}^{b}(y)\right| \Psi\right\rangle=\frac{1}{2} \delta^{a b} t_{i j}(x) \omega^{-1}(x, y)
$$

with $t_{i j}$ being the transverse projector. It is determined by solving the functional Schrödinger equation, i.e. minimizing the energy $\langle\Psi|H| \Psi\rangle$. This gives rise to a non-linear integral equation in $\omega$ which we refer to as the gap equation. It was derived to two-loop order in the energy in Ref. [10] and reads in momentum space $(k=|\mathbf{k}|)$

$$
\omega^{2}(k)=k^{2}+\chi^{2}(k)+I_{\omega}(k)+I_{\omega}^{0} .
$$

Here, $\chi(k)$ abbreviates the so-called curvature and it is related by

$$
\chi(k)=\frac{N_{c}}{4} \int \frac{d^{3} q}{(2 \pi)^{3}}\left(1-(\hat{\mathbf{k}} \cdot \hat{\mathbf{q}})^{2}\right) \frac{d(|\mathbf{k}-\mathbf{q}|) d(q)}{(\mathbf{k}-\mathbf{q})^{2}}
$$

to the ghost propagator

$$
\left\langle\Psi\left|(-D \partial)^{-1}\right| \Psi\right\rangle=\frac{1}{g} \frac{d(k)}{k^{2}} .
$$

A Dyson-Schwinger equation for the ghost form factor $d$ may be derived from the path integral, or alternatively from the following operator identity for $G[A]=(-D \partial)^{-1}$,

$$
G[A]=\left(-\partial^{2}\right)^{-1}+\left(-\partial^{2}\right)^{-1} g \hat{A} \partial G[A]
$$

which yields

$$
d^{-1}(k)=g^{-1}-\frac{N_{c}}{2} \int \frac{d^{3} q}{(2 \pi)^{3}}\left(1-(\hat{\mathbf{k}} \cdot \hat{\mathbf{q}})^{2}\right) \frac{d(|\mathbf{k}-\mathbf{q}|)}{(\mathbf{k}-\mathbf{q})^{2} \omega(q)} .
$$


Both in the ghost Dyson-Schwinger equation (2.10) and in the equation for the curvature (2.7), we have approximated the proper ghost-gluon vertex by its tree-level counterpart $\Gamma_{i}^{0}$. This amounts to the factorization

$$
\left\langle A_{i} G[A]\right\rangle=D_{i j}\left\langle G[A] \Gamma_{j}^{0} G[A]\right\rangle \approx D_{i j}\langle G[A]\rangle \Gamma_{j}^{0}\langle G[A]\rangle .
$$

The non-renormalization of the ghost-gluon vertex in gauges where the gluon propagator is transverse, such as the Coulomb and the Landau gauge [16, 17], suggests that the above approximation is a good one. Dyson-Schwinger studies in both four and three-dimensional Landau gauge as well as lattice calculations in four-dimensional Landau gauge confirmed that the dressed vertex is close to tree-level [18]. The case of three-dimensional Landau gauge resembles the Coulomb gauge and therefore we adopt the approximation (2.11). This vertex' non-renormalization will have crucial impact on the IR sector of the solutions.

The other momentum dependent term $I_{\omega}(k)$ in the gap equation (2.6) reads

$$
I_{\omega}(k)=\frac{N_{C}}{4} \int \frac{d^{3} q}{(2 \pi)^{3}}\left(1+(\hat{\mathbf{k}} \cdot \hat{\mathbf{q}})^{2}\right) \frac{d(\mathbf{k}-\mathbf{q})^{2} f(\mathbf{k}-\mathbf{q})}{(\mathbf{k}-\mathbf{q})^{2}} \frac{[\omega(\mathbf{q})-\chi(\mathbf{q})+\chi(\mathbf{k})]^{2}-\omega(\mathbf{k})^{2}}{\omega(\mathbf{q})}
$$

and is due to the Coulomb interaction part of the Hamiltonian. Here, the form factor $f$ measures the deviation from the factorization of the Coulomb potential,

$$
\left\langle G[A]\left(-\partial^{2}\right) G[A]\right\rangle=\langle G[A]\rangle\left(-\partial^{2}\right) f\langle G[A]\rangle .
$$

In the infrared, we set $f(k)=1$, factorizing the expectation value for the Coulomb propagator (2.13) equivalently to the one for the ghost-gluon vertex in Eq. (2.11). In the ultraviolet, $f(k)$ is treated perturbatively, see [10].

In order to fix the Coulomb gauge uniquely, configuration space must be restricted to the compact fundamental modular region. As suggested in [8], this entails the "horizon condition" for the ghost form factor,

$$
d^{-1}(0)=0 \text {. }
$$

As we shall see, the horizon condition (2.14) has the consequence that all form factors $d, \chi$ and $\omega$ diverge in the infrared.

\section{Green functions, heavy quark potential and running coupling}

The ultraviolet divergences encountered in the gap equation (2.6) are removed by subtracting the equations at an arbitrary renormalization scale $\mu$. Alternatively, one can eliminate the divergences by adding appropriate counter terms to the YM Hamiltonian and to $\ln \mathscr{J}$ [19]. This eliminates the UV-divergent constant $I_{\omega}^{0}$ from Eq. (2.6) and involves some renormalization constants, one of them can be chosen as $c=\lim _{k \rightarrow 0}(\omega(k)-\chi(k))$ and fixed by the requirement of minimal energy to be $c=0$. For details, see Ref. [20].

The solutions for the form factors $\omega(k), d(k)$ and $\chi(k)$ can be seen in Fig. 1. In the asymptotic infrared, the gluon form factor $\omega(k)$ approaches the curvature $\chi(k)$, reflecting the dominance of ghost degrees of freedom, cf. Landau gauge [4]. With $\chi(k)$ being infrared enhanced, the ghost content of the solution makes propagation of gluons over asymptotically large distances impossible, hence gluons are confined. 

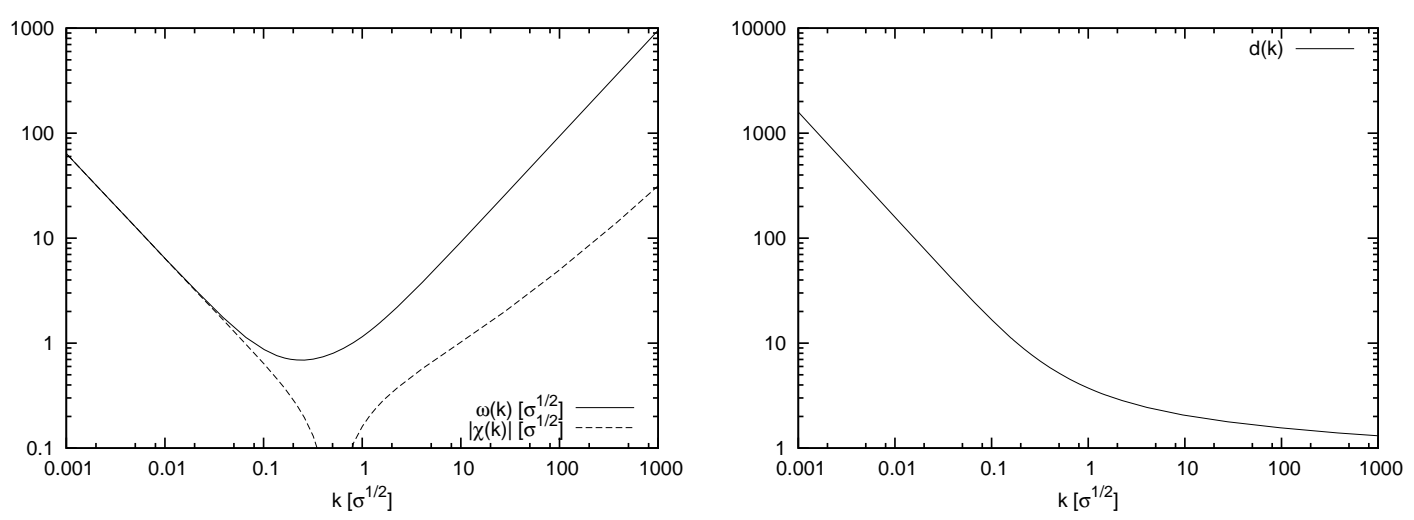

Figure 1: Left: the gluon form factor $\omega(k)$ and the curvature $|\chi(k)|$. Right: the ghost form factor $d(k)$.

The correlation of the asymptotic infrared power laws is due to the non-renormalization of the ghost-gluon vertex,

$$
\omega(k)=\chi(k) \sim d(k) \sim \frac{1}{k}, f(k)=1, \quad k \rightarrow 0 .
$$

Without imposing the horizon condition (2.14), solutions to $d, \chi$ and $\omega$ can be found that approach finite values in the infrared [19] so that the energy functional is dominated by the ultraviolet modes, as speculated by Feynman [13]. Conversely, the infrared power law solution (3.1) where the horizon condition is satisfied are not subdominant to ultraviolet modes and turn out independently of the details of the wave functional. Even a stochastic vacuum, $\Psi[A]=1$, would produce the same results for the infrared [15]. One may therefore be confident using the variational principle.

The infrared enhancement of the form factors $\omega(k)$ and $d(k)$ is qualitatively reproduced by recent lattice calculations [21].

Equipped with the ghost form factor $d(k)$, the heavy quark potential can be found by choosing

$$
\rho_{m}^{a}(x)=\delta^{a 3}\left(\delta^{(3)}(x-r / 2)-\delta^{(3)}(x+r / 2) .\right)
$$

and recalculating the energy $\langle H\rangle$ with fixed $\omega$. There is only one contribution to the energy that depends on the distance $r$ between the quarks. Using Eq. (2.13), it reads

$$
V_{c}(r)=\frac{g^{2}}{2} \int\left\langle\Psi\left|\rho_{m} F \rho_{m}\right| \Psi\right\rangle=\int \frac{d^{3} q}{(2 \pi)^{3}} \frac{d^{2}(q) f(q)}{q^{2}}\left(1-e^{i \mathbf{q} \cdot \mathbf{r}}\right) .
$$

With the infrared behavior of the form factors (3.1), we find that $V_{c}(r)$ rises linearly in the infrared and thus confines heavy quarks as shown in Fig. 2. By matching the slope of the linear potential to the lattice string tension $\sigma$, one may set the scale.

Apart from the solution in Fig. 1 with the asymptotic infrared behavior (3.1) there is one further solution with slightly different infrared exponents for the power laws. The latter was discovered first [10], however, it does not have the same attractive features as the one in Fig. 1. In particular, the heavy quark potential is strictly linearly rising only for the solution presented here.

A nonperturbative running coupling may be extracted from the ghost-gluon vertex [17, 11],

$$
\alpha(k)=\frac{2}{3 \pi} k d^{2}(k) \omega^{-1}(k),
$$



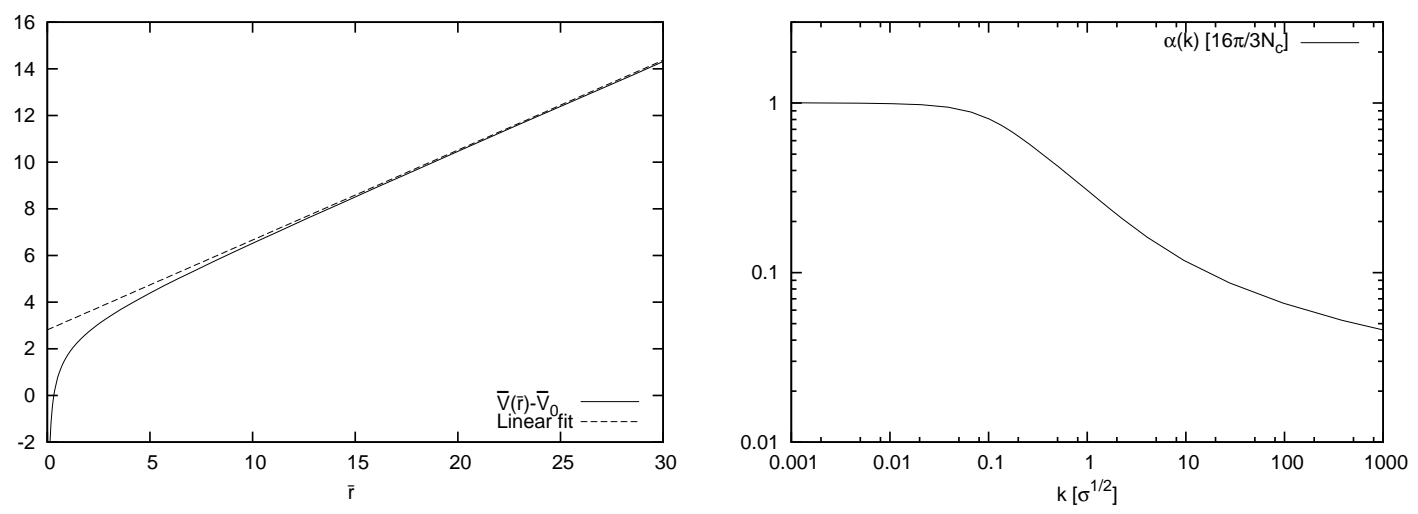

Figure 2: Right: the Coulomb potential $V(r)$. Right: the running coupling $\alpha(k)$.

With a tree-level vertex, it can be shown that one finds a finite value in the infrared, $\alpha(0)=$ $16 \pi /\left(3 N_{c}\right)$ [11]. In the ultraviolet, we find the correct $1 / \ln (k / \mu)$ scaling from one-loop perturbation theory. However, the first coefficient of the beta function, $\beta_{0}$, is off by a factor of $8 / 11$. This is due to the approximations and requires further investigation. Recent lattice calculations in Coulomb gauge [22] show qualitative agreement with the running coupling presented here. In comparison to the analogous running coupling in the Landau gauge [4], we do not find a bump for intermediate momenta yielding a spurious zero in the beta function. Note that $\alpha(k)$ in Fig. 2 is a monotonic function.

\section{The 't Hooft loop}

A (dis-)order parameter of confinement is the 't Hooft loop $\langle V(C)\rangle$ [23] whose operator $V(C)$ is defined by the relation $V\left(C_{1}\right) W\left(C_{2}\right)=Z^{L\left(C_{1}, C_{2}\right)} W\left(C_{2}\right) V\left(C_{1}\right)$, where $W(C)$ is the operator of the spatial Wilson loop, $Z$ is a (non-trivial) center element of the gauge group and $L\left(C_{1}, C_{2}\right)$ denotes the Gaussian linking number. An explicit realization of $V(C)$ in continuum Yang-Mills theory was derived in ref. [24] and is given by

$$
V(C)=\exp \left[i \int d^{3} x \mathscr{A}_{i}^{a}[C](x) \Pi_{i}^{a}(x)\right] .
$$

Here $\mathscr{A}[C]$ denotes the gauge potential of a (spatial) center vortex whose magnetic flux is localized at the loop $C$. Since $V(C) \Psi(A)=\Psi(A+\mathscr{A}[C])$ the 't Hooft loop is a center vortex generator. Using the wave functional found in the variational solution of the Yang-Mills Schrödinger equation in Coulomb gauge, as described above, the expectation value $\langle V(C)\rangle \equiv \exp (-S(C))$ was evaluated for a planar circular loop $C$ and is was found that the exponential $S(C)$ obeys a perimeter law signaling confinement [20]. This result is in accord with the linear behavior found for the static color potential.

\section{Conclusions}

We have solved the Yang-Mills Schrödinger equation approximately and thus determined the vacuum wave functional. Our solutions exhibit the phenomena of confinement of gluons as well 
as heavy quarks. As an improvement on previous results, the heavy quark potential rises strictly linearly. The nonperturbative running coupling derived from the ghost-gluon vertex was presented and the 't Hooft loop was calculated. It is promising that the results have the crucial features of nonperturbative physics, and that calls for further investigations in the Hamiltonian approach.

\section{Acknowledgments}

It is a pleasure to thank A. Szczepaniak and A. Weber for continuing correspondence on the subject. One of us (H.R.) would like to thank the organizers of the conference for the stimulating event.

\section{References}

[1] J. Greensite, Prog. Part. Nucl. Phys. 51 (2003) 1 [arXiv:hep-lat/0301023]; G. S. Bali, Phys. Rept. 343 (2001) 1 [arXiv:hep-ph/0001312].

[2] C. S. Fischer, R. Alkofer and H. Reinhardt, Phys. Rev. D 65 (2002) 094008 [arXiv:hep-ph/0202195]; C. S. Fischer, A. Maas, J. M. Pawlowski and L. von Smekal, arXiv:hep-ph/0701050.

[3] L. von Smekal, R. Alkofer and A. Hauck, Phys. Rev. Lett. 79 (1997) 3591 [arXiv:hep-ph/9705242]; L. von Smekal, A. Hauck and R. Alkofer, Annals Phys. 267 (1998) 1 [Erratum-ibid. 269 (1998) 182] [arXiv:hep-ph/9707327].

[4] C. S. Fischer, J. Phys. G 32 (2006) R253 [arXiv:hep-ph/0605173], and references therein.

[5] P. Watson and H. Reinhardt, arXiv:0709.3963 [hep-th], arXiv:0709.0140 [hep-th]; Phys. Rev. D 75 (2007) 045021 [arXiv:hep-th/0612114].

[6] I. B. Khriplovich, Yad. Fiz. 10 (1969) 409.

[7] V. N. Gribov, Nucl. Phys. B 139 (1978) 1.

[8] D. Zwanziger, Nucl. Phys. B 485 (1997) 185 [arXiv:hep-th/9603203].

[9] A. P. Szczepaniak and E. S. Swanson, Phys. Rev. D 65 (2001) 025012 [arXiv:hep-ph/0107078]; A. P. Szczepaniak, Phys. Rev. D 69 (2004) 074031 [arXiv:hep-ph/0306030].

[10] C. Feuchter and H. Reinhardt, Phys. Rev. D 70 (2004) 105021 [arXiv:hep-th/0408236], arXiv:hep-th/0402106.

[11] W. Schleifenbaum, M. Leder and H. Reinhardt, Phys. Rev. D 73 (2006) 125019 [arXiv:hep-th/0605115].

[12] D. Epple, H. Reinhardt and W. Schleifenbaum, Phys. Rev. D 75 (2007) 045011 [arXiv:hep-th/0612241].

[13] R. P. Feynman, in *Wangerooge 1987, proceedings, Variational Calculations in Quantum Field Theory*, p. 28-40.

[14] N. H. Christ and T. D. Lee, Phys. Rev. D 22 (1980) 939 [Phys. Scripta 23 (1981) 970].

[15] H. Reinhardt and C. Feuchter, Phys. Rev. D 71 (2005) 105002 [arXiv:hep-th/0408237].

[16] J. C. Taylor, Nucl. Phys. B 33 (1971) 436;

[17] C. S. Fischer and D. Zwanziger, Phys. Rev. D 72 (2005) 054005 [arXiv:hep-ph/0504244].

[18] A. Cucchieri, T. Mendes and A. Mihara, JHEP 0412 (2004) 012 [arXiv:hep-lat/0408034]; W. Schleifenbaum, A. Maas, J. Wambach and R. Alkofer, Phys. Rev. D 72 (2005) 014017 [arXiv:hep-ph/0411052]; A. Sternbeck, E. M. Ilgenfritz, M. Muller-Preussker and A. Schiller, PoS LAT2005 (2006) 333 [arXiv:hep-lat/0509090].

[19] D. Epple, H. Reinhardt, W. Schleifenbaum and A. P. Szczepaniak, arXiv:0712.3694 [hep-th].

[20] H. Reinhardt and D. Epple, Phys. Rev. D 76 (2007) 065015 [arXiv:0706.0175 [hep-th]].

[21] M. Quandt, PoS LAT2007 325; A. Voigt, E. M. Ilgenfritz, M. Mueller-Preussker and A. Sternbeck, arXiv:0709.4585 [hep-lat], PoS LAT2007 338.

[22] S. Furui and H. Nakajima, arXiv:0708.1421 [hep-lat], PoS LAT2007 301.

[23] G. 't Hooft, Nucl. Phys. B 138, 1 (1978).

[24] H. Reinhardt, Phys. Lett. B 557, 317 (2003) [arXiv:hep-th/0212264]. 